\title{
Symmetric Vertex Models on Planar Random Graphs
}

\author{
D.A. Johnston \\ Dept. of Mathematics \\ Heriot-Watt University \\ Riccarton \\ Edinburgh, EH14 4AS, Scotland
}

August 20, 2018

\begin{abstract}
We solve a 4-(bond)-vertex model on an ensemble of 3-regular $\left(\Phi^{3}\right)$ planar random graphs, which has the effect of coupling the vertex model to $2 \mathrm{D}$ quantum gravity. The method of solution, by mapping onto an Ising model in field, is inspired by the solution by Wu et.al. of the regular lattice equivalent - a symmetric 8-vertex model on the honeycomb lattice, and also applies to higher valency bond vertex models on random graphs when the vertex weights depend only on bond numbers and not cyclic ordering (the so-called symmetric vertex models).

The relations between the vertex weights and Ising model parameters in the 4-vertex model on $\Phi^{3}$ graphs turn out to be identical to those of the honeycomb lattice model, as is the form of the equation of the Ising critical locus for the vertex weights. A symmetry of the partition function under transformations of the vertex weights, which is fundamental to the solution in both cases, can be understood in the random graph case as a change of integration variable in the matrix integral used to define the model.

Finally, we note that vertex models, such as that discussed in this paper, may have a role to play in the discretisation of Lorentzian metric quantum gravity in two dimensions.
\end{abstract}




\section{Introduction}

Random matrix models have proved remarkably successful in investigating the critical behaviour of various sorts of spin models (Ising [1, 2], Potts [3], $O(N)$ [4] ...) living on ensembles of planar random graphs. This, in effect, couples the models to $2 D$ quantum gravity which originally motivated their study in the context of string theory and random surfaces [5]. From the purely statistical mechanical point of view one is looking at the critical behaviour of an (annealed) ensemble of random graphs decorated by the spins. The connection with gravity comes from the fact that the graphs can adapt their connectivity in response to the spin configuration, which in turn is influenced by the connectivity of the graph on which it lives. This captures the essential feature of the back reaction of the matter on the geometry that characterises gravity in a discrete form.

The graphs of interest appear as planar Feynman diagrams in the perturbation expansion in the vertex coupling of a Hermitian matrix model free energy of the general form [6]

$$
F=\frac{1}{M^{2}} \log \int \prod_{i} D \Phi_{i} \exp (-S)
$$

in the limit $M \rightarrow \infty$, where the $\Phi_{i}$ are sufficient $M \times M$ Hermitian matrix variables to describe the matter decoration and the log ensures that only connected graphs contribute. For the Ising model on trivalent planar graphs, for instance, we need two matrices $X, Y$ and the action takes the form

$$
S=\operatorname{Tr}\left\{\frac{1}{2}\left(X^{2}+Y^{2}\right)-g X Y-\frac{\lambda}{3}\left[e^{h} X^{3}+e^{-h} Y^{3}\right],\right\}
$$

where $g=\exp (-2 \beta)$ and $h$ is the external field. We can see that the $X^{3}$ terms can be thought of as spin-up or + vertices and the $Y^{3}$ terms spin-down or - , while the inverse of the quadratic terms gives the appropriate edge weights for,+++- and -- edges.

To date there has been less consideration of arrow vertex models on ensembles of planar random graphs and none at all of bond vertex models. In the arrow case one decorates the edges of the graph with arrows and assigns weights to the vertices depending on the local arrow configurations around each vertex, in the bond case one simply has empty or occupied edges (bonds) and vertex weights depending on the local bond configuration. Some arrow vertex models have been discussed using elementary means in [7, 8], mostly on 4-valent random graphs, revealing $X Y$ and Ising critical behaviour for suitable coupling constant loci. More recently some elegant and sophisticated work using character expansions [9] has provided a full solution of an arrow vertex model on 4-valent planar graphs confirming the $X Y$ critical point found in [7].

In this paper we give the first solutions of bond vertex models on planar random graphs. Our main example is a 4-vertex model on $\Phi^{3}$ random graphs, but the general method works for any bond vertex models whose weights depend only on the number of bonds at a vertex rather than their particular cyclic ordering. On regular lattices such models are termed symmetric vertex models and we retain the nomenclature here. Since our 4-vertex model on $\Phi^{3}$ random graphs is the random graph equivalent of the symmetric 8-vertex model on the honeycomb lattice discussed by Wu et.al. [10, 11, 12] we take our cue from [10] where the honeycomb lattice model was first solved by making use of the so-called generalised weak graph transformation [13] between vertex weights. This brought the partition function to a form that was recognisable as that of an Ising model in field an the critical behaviour of the model was then deduced from the corresponding Ising results.

In what follows we first define our 4-vertex model, then solve it by mapping onto the Ising model in field on $\Phi^{3}$ planar random graphs, which itself was solved in [1]. We move on to discuss higher valency symmetric models and show that the same general method of solution still works. We then compare these results with those of 10, 11, 12] for the 8-vertex model on the honeycomb lattice and note that the generalized weak graph symmetry of the honeycomb lattice model finds a counterpart in an orthogonal variable transformation in the matrix integrals used to define the random graph models. Finally, we close

with some speculations on the relations of vertex models to Lorentzian metric quantum gravity, where they can be thought of as providing a discretisation of causal structure. 


\section{The 4-Vertex Model on 3-valent random graphs}

The canonical (fixed number of vertex) partition function for the 4-vertex model on $\Phi^{3}$ planar random graphs is given by

$$
Z_{4 v}=Z_{4 v}(a, b, c, d)=\sum_{\Phi_{N}} \sum_{G} a^{N_{1}} b^{N_{2}} c^{N_{3}} d^{N_{4}}
$$

where there are two summations: $\sum_{\Phi_{N}}$ over $\Phi^{3}$ random graphs with $N$ vertices and $\sum_{G}$ over all possible bond configurations on each $\Phi^{3}$ graph built using the vertices of Fig.1. Each graph has $N_{1}$ vertices of type (a), $N_{2}$ of type (b), $N_{3}$ of type (c), $N_{4}$ of type $(d)$ and $N=N_{1}+N_{2}+N_{3}+N_{4}$.

We can generate the graphs we require, suitably decorated with the allowed bond configurations, by inserting the action

$$
S=\operatorname{Tr}\left\{\frac{1}{2}\left(X^{2}+Y^{2}\right)-\lambda\left[\frac{a}{3} X^{3}+\frac{d}{3} Y^{3}+c X Y^{2}+b X^{2} Y\right]\right\} .
$$

into equ.(1D), where $X, Y$ are $M \times M$ Hermitian matrices and we have introduced an overall vertex coupling $\lambda$ for convenience.. The limit $M \rightarrow \infty$ picks out the planar diagrams. A direct attempt at solving a matrix model with the action equ.(画) might be possible using the character expansion techniques of [9], but it turns out to be easier to follow in the footsteps of [10, 11] and establish a correspondence with an Ising model in order to determine the critical behaviour of the vertex model.

We start by by carrying out the orthogonal transformations

$$
\begin{aligned}
& X \rightarrow(X+Y) / \sqrt{2} \\
& Y \rightarrow(X-Y) / \sqrt{2}
\end{aligned}
$$

on the matrices in the Ising action of equ.(2) , followed by the rescalings $X \rightarrow X /(1-g)^{1 / 2}, Y \rightarrow$ $Y /(1+g)^{1 / 2}, \lambda \rightarrow \sqrt{2} \lambda(1-g)^{3 / 2}$. This change of variable in the matrix integral has a trivial Jacobian and gives the new action

$$
S=\operatorname{Tr}\left\{\frac{1}{2}\left(X^{2}+Y^{2}\right)-\frac{\lambda \cosh (h)}{3}\left[X^{3}+3 g^{*} X Y^{2}\right]-\frac{\lambda \sinh (h)\left(g^{*}\right)^{3 / 2}}{3}\left[Y^{3}+\frac{3}{g^{*}} X^{2} Y\right]\right\} .
$$

where $g^{*}=(1-g) /(1+g)$, which is clearly of the same general form as equ.(1). The bond vertex weights from equ.(6) are also given in Fig.1. It should be remarked that the transformation of equ.(55) is the duality transformation for the Ising model on random $\Phi^{3}$ graphs [14], and that the bond graphs generated by the transformed action in equ.(6) are precisely the high-temperature expansion graphs of the original Ising model on $\Phi^{3}$ graphs.

We can see that the vertex weights coming from the Ising model in equ.(6), which we denote by tilde'd quantities,

$$
\begin{aligned}
\tilde{a} & =\cosh (h) \\
\tilde{b} & =\sinh (h)\left(g^{*}\right)^{1 / 2} \\
\tilde{c} & =\cosh (h) g^{*} \\
\tilde{d} & =\sinh (h)\left(g^{*}\right)^{3 / 2}
\end{aligned}
$$

are fixed in the ratio $\tilde{a} \tilde{d}=\tilde{b} \tilde{c}$, which will not be the case for the generic weights of equ.(4). However, if we consider the effect of the further orthogonal rotation 1

$$
\left(\begin{array}{c}
X \\
Y
\end{array}\right) \rightarrow\left(\begin{array}{cc}
\cos (\theta) & \sin (\theta) \\
-\sin (\theta) & \cos (\theta)
\end{array}\right)\left(\begin{array}{c}
X \\
Y
\end{array}\right)
$$

\footnotetext{
${ }^{1}$ This preserves the propagator $X^{2}+Y^{2}$ in the action of equ.(6) and again has a trivial Jacobian, so it does not effect the evaluation of the matrix integral.
} 
on the vertex weights of equ.(4), we find

$$
\begin{aligned}
\tilde{a} & =\frac{1}{\left(1+y^{2}\right)^{3 / 2}}\left[a+3 y b+3 y^{2} c+y^{3} d\right] \\
\tilde{b} & =\frac{1}{\left(1+y^{2}\right)^{3 / 2}}\left[-y a+\left(1-2 y^{2}\right) b-\left(y^{3}-2 y\right) c+y^{2} d\right] \\
\tilde{c} & =\frac{1}{\left(1+y^{2}\right)^{3 / 2}}\left[y^{2} a+\left(y^{3}-2 y\right) b+\left(1-2 y^{2}\right) c+y d\right] \\
\tilde{d} & =\frac{1}{\left(1+y^{2}\right)^{3 / 2}}\left[-y^{3} a+3 y^{2} b-3 y c+d\right]
\end{aligned}
$$

where we have extracted $\cos (\theta)$, denoted $\tan (\theta)$ as $y$ and deliberately used tilde'd quantities again for the transformed weights. We can now demand that these transformed weights satisfy $\tilde{a} \tilde{d}=\tilde{b} \tilde{c}$, just as in equ.(6) which has the effect of making the partition function of the vertex model equivalent to that of the Ising model in field. Setting $\tilde{a} \tilde{d}=\tilde{b} \tilde{c}$ gives the following equation for the rotation parameter $y$ from equating the right hand sides of equ.(9)

$$
B y^{2}+2(C-A) y-B=0
$$

where $A=b d-c^{2}, B=a d-b c, C=a c-b^{2}$. Since $-\infty \leq y \leq \infty$ is at our disposal we can solve this for general $a, b, c, d$ to obtain transformed weights satisfying $\tilde{a} \tilde{d}=\tilde{b} \tilde{c}$.

To deduce the critical behaviour of the vertex model by using this correspondence with the Ising model in field we need the relation between the vertex weights and the Ising parameters $\beta$ and $h$. The Ising and tilde'd vertex model parameters were related by

$$
\begin{aligned}
\tanh (\beta) & =\frac{\tilde{c}}{\tilde{a}} \\
\tanh (h) & =\frac{\tilde{b}}{\sqrt{\tilde{a} \tilde{c}}} .
\end{aligned}
$$

as can be seen directly from equ.(6). We can then substitute for the tilde'd weights in terms of the original weights and $y$ using equ. $(9)$. to give the relation between the original weights $a, b, c, d$ and the Ising parameters $\beta, h$

$$
\begin{aligned}
\exp (2 \beta) & =\frac{B y+C-A}{A+C} \\
\tanh (h) & =\frac{W}{T}\left(\frac{B y+2 C}{B y-2 A}\right)^{1 / 2},
\end{aligned}
$$

where $y$ is again the one of the solutions of equ.110), $A, B, C$ are as above, $T=(b+d) y+a+c$ and $W=(b+d)-(a+c) y$.

On $\Phi^{3}$ random graphs the Ising model displays a third order magnetisation transition in zero field. The second of equs.(12) shows that zero Ising field implies that $W=0$, which in terms of the vertex weights (when $y$ is substituted for) gives the locus

$$
a\left(b^{3}+d^{3}\right)-d\left(a^{3}+c^{3}\right)+3(a b+b c+c d)\left(c^{2}-b d-b^{2}+a c\right)=0 .
$$

From the vertex model perspective it is most natural to disallow loops of length one and two in the random graphs in order to make sure that there are no double edges or tadpoles, in which case the Ising critical temperature is given by $\beta_{c}=\frac{1}{2} \log \left(\frac{103}{28}\right)=0.7733185$ [2]. The first of equs.(12) then gives

$$
\frac{103}{28}=\frac{\sqrt{\left(a c-b^{2}-b d+c^{2}\right)^{2}+(a d-b c)^{2}}}{a c-b^{2}+b d-c^{2}}
$$

as the position of the third order critical point on the locus equ.(13). We still have, of course, the echo of the field driven Ising transition appearing for $\beta>\beta_{c}$ along the locus equ.(13). 


\section{Higher valency vertex models}

In this section we show that on 4 -valent $\left(\Phi^{4}\right)$ random graphs the Ising equivalency can still be easily established by rotating the variables in a matrix model action. One extra ingredient in the 4 -valent case compared with the 3 -valent case is that the cyclic order of the bonds allows one to distinguish between two different sorts of vertices with two occupied and two empty edges, one with occupied bonds at right angles and one with occupied bonds "straight through". The Ising equivalence only holds for the symmetric case in which equal weights are assigned to these bond configurations.

On $\Phi^{4}$ random graphs the Ising model action is

$$
S=\operatorname{Tr}\left\{\frac{1}{2}\left(X^{2}+Y^{2}\right)-g X Y+\frac{\lambda}{4}\left[e^{h} X^{4}+e^{-h} Y^{4}\right],\right\}
$$

with only a higher order potential distinguishing the action from that for $\Phi^{3}$ graphs in equ.(2). The change of variables $X \rightarrow(X+Y) / \sqrt{2}, Y \rightarrow(X-Y) / \sqrt{2}$ and rescalings $X \rightarrow X /(1-g)^{1 / 2}, Y \rightarrow Y /(1+g)^{1 / 2}$, $\lambda \rightarrow 2 \lambda(1-g)^{2}$ gives the action

$$
\begin{aligned}
S & =\operatorname{Tr}\left\{\frac{1}{2}\left(X^{2}+Y^{2}\right)+\frac{\lambda \cosh (h)}{4}\left[X^{4}+\left(g^{*}\right)^{2} Y^{4}\right]+\frac{\lambda \cosh (h) g^{*}}{2}\left[2 X^{2} Y^{2}+X Y X Y\right]\right. \\
& \left.+\lambda \sinh (h) g^{*}\left[\sqrt{g^{*}} X Y^{3}+\frac{1}{\sqrt{g^{*}}} X^{3} Y\right]\right\}
\end{aligned}
$$

where, as on $\Phi^{3}$ graphs, $g^{*}=(1-g) /(1+g)$. Note the presence of the $\left[2 X^{2} Y^{2}+X Y X Y\right]$ term fixing the weights of right angled and straight through two-bond vertices to be the same. It should be remarked once again that the change of variables in equ.(5) is a duality transformation for the model, and that equ.(16) generates the high temperature expansion graphs of the original Ising model in equ.(15).

The effect of the orthogonal rotation of equ.(8) on the vertices arranged in some suitable lexicographic order (e.g. $X^{4}, X^{3} Y, X^{2} Y^{2}, X Y^{3}, Y^{4}$ as labelled on Fig.2) gives

$$
\begin{aligned}
\tilde{a} & =\frac{1}{\left(1+y^{2}\right)^{2}}\left[a+4 y b+6 y^{2} c+4 y^{3} d+y^{4} e\right] \\
\tilde{b} & =\frac{1}{\left(1+y^{2}\right)^{2}}\left[-y a+\left(1-3 y^{2}\right) b-\left(3 y^{3}-3 y\right) c+\left(3 y^{2}-y^{4}\right) d+y^{3} e\right] \\
\tilde{c} & =\frac{1}{\left(1+y^{2}\right)^{2}}\left[y^{2} a+\left(2 y^{3}-2 y\right) b+\left(y^{4}-4 y^{2}+1\right) c+\left(2 y-2 y^{3}\right) d+y^{2} e\right] \\
\tilde{d} & =\frac{1}{\left(1+y^{2}\right)^{2}}\left[-y^{3} a+\left(3 y^{2}-y^{4}\right) b-\left(3 y-3 y^{3}\right) c+\left(1-3 y^{2}\right) d+y e\right] \\
\tilde{e} & =\frac{1}{\left(1+y^{2}\right)^{2}}\left[y^{4} a-4 y^{3} b+6 y^{2} c-4 y d+e\right] .
\end{aligned}
$$

The strategy for solving the $\Phi^{4}$ model is identical to the $\Phi^{3}$ case. One uses the above transformations of the vertex weights to bring the model to the Ising action defined above in equ.(16). The tilde'd weights in this $\left(\tilde{a}\right.$ for $X^{4}$ etc.) can be characterised in various ways, for example: $\tilde{a} \tilde{d}=\tilde{d} \tilde{c}, \tilde{c}^{2}=\tilde{a} \tilde{e}$. Having achieved this the critical behaviour can again be read off from that of the Ising model in field, this time on $\Phi^{4}$ graphs. Indeed, it is clear that generic symmetric vertex models on random graphs can be solved by using this method. In the q-valent case one carries out the same sequence of two orthogonal rotations on the action for the Ising model on $\Phi^{q}$ planar random graphs

$$
S=\operatorname{Tr}\left\{\frac{1}{2}\left(X^{2}+Y^{2}\right)-g X Y+\frac{\lambda}{q}\left[e^{h} X^{q}+e^{-h} Y^{q}\right]\right\} .
$$

to obtain the action for the symmetric vertex model. One can thus deduce that the critical behaviour of the symmetric vertex model on q-valent random graphs will be Ising-like. 


\section{Comparison with the Symmetric 8-Vertex Model on the hon- eycomb lattice}

The closest regular lattice equivalent of our $\Phi^{3}$ planar random graphs is the honeycomb lattice where each vertex still has coordination number three but, in contrast to the random graphs, all loops are of length six. The eight possible bond vertex configurations all look similar to those for the random lattice in Fig.1 but we now have orientational order as well as cyclic order around each vertex so $b$ and $c$ represent three possible orientations each. However if we impose equality between the different orientations, we arrive at the symmetric vertex model of [10, 11, 12 which, like the $\Phi^{3}$ random graph vertex model, has 4 distinct vertex weights. The partition function is given in a similar fashion to equ.(3) by

$$
Z_{8 v}=Z_{8 v}(a, b, c, d)=\sum_{G} a^{N_{1}} b^{N_{2}} c^{N_{3}} d^{N_{4}}
$$

where there is now no sum over random graphs since all the bond configurations are living on the honeycomb lattice.

Several symmetry properties of the partition function are immediately apparent. From the negation of vertices occurring in pairs we have $Z_{8 v}(a, b, c, d)=Z_{8 v}(-a,-b,-c,-d)=Z_{8 v}(-a, b,-c, d)=$ $Z_{8 v}(a,-b, c,-d)$. Similarly, exchanging dark and light bonds gives $Z_{8 v}(a, b, c, d)=Z_{8 v}(d, c, b, a)$. Perhaps rather less obvious is the generalised weak graph symmetry 13] $Z_{8 v}(a, b, c, d)=Z_{8 v}(\tilde{a}, \tilde{b}, \tilde{c}, \tilde{d})$, where the transformations between the original and tilde'd variables are precisely those generated by the orthogonal rotations in the random graph model in equ.(9).

In 11] the generalised weak graph symmetry on the honeycomb lattice was thought of as being generated by rotations with

$$
V(y)=\frac{1}{\left(1+y^{2}\right)^{1 / 2}}\left(\begin{array}{cc}
1 & y \\
y & -1
\end{array}\right)
$$

on the vertices in "bond space", where occupied and empty bonds on a given edge were to be thought of as components of a vector. An alternative choice of transformation

$$
U(y)=\frac{1}{\left(1+y^{2}\right)^{1 / 2}}\left(\begin{array}{cc}
1 & y \\
-y & 1
\end{array}\right)
$$

was related to $V(y)$ by the negations $\tilde{b} \rightarrow-\tilde{b}, \tilde{d} \rightarrow-\tilde{d}$ and was hence not independent. Remarkably, the second transformation is just that generated by equ.(8) in the random graph model.

The generalised weak graph symmetry played an important role in the original solution of the honeycomb lattice model [11] because a suitable choice of $y$ allowed generic vertex weights $a, b, c, d$ to be transformed to $\tilde{a}, \tilde{b}, \tilde{c}, \tilde{d}$ satisfying $\tilde{a} \tilde{d}=\tilde{b} \tilde{c}$ just as for the random lattice. For vertex weights satisfying the latter condition

$$
Z_{8 v}(\tilde{a}, \tilde{b}, \tilde{c}, \tilde{d})=(\tilde{a} \cosh (h) / 2)^{N}(\cosh (\beta))^{-3 N / 2} Z_{\text {Ising }}(\beta, h)
$$

where $Z_{\text {Ising }}$ was the standard honeycomb lattice Ising partition function. The equivalence followed from observing that this choice of weights meant that the vertices generated precisely the right weights for the diagrams of the high temperature expansion of the Ising model on the honeycomb lattice.

The critical behaviour of the symmetric 8-vertex model on the honeycomb lattice was thus deduced by following exactly the same path we have taken for the 4-vertex model on $\Phi^{3}$ random graphs - exploiting a symmetry of the partition function to transform it to that of an Ising model in field. The parallels run even closer since equs.(9, 10, 11, 12, 13) still hold identically for the honeycomb lattice vertex model and the only difference in equ.(14) is in the numerical value of the Ising critical temperature on the left hand side. This might grandiloquently be phrased as a non-renormalization theorem for the vertex weights since the ratios of weights in equ.(11) are unaffected by the coupling to gravity that is represented by the sum over random graphs.

The close correspondence between the $\Phi^{3}$ and honeycomb results becomes a little less surprising when one considers a second determination of the critical behaviour of the honeycomb lattice model by Wu in 
[12] where it was shown that a direct mapping between the Ising and vertex models was possible when a decoration-iteration transformation was employed. In Fig.3 we show a single vertex, which hosts a spin subject to an external field $H$. In addition the edges have spins which are subject to a field $2 H^{\prime}$ and the two sorts of spins interact via a spin-spin coupling $R$. The presence of a dark bond can be denoted by an edge spin $\sigma=1$ and its absence by $\sigma=-1$. The vertex model weights can then be represented correctly in terms of appropriate $H^{\prime}, H$ and $R$

$$
\begin{aligned}
\cosh (2 R) & =\frac{B}{2(A C)^{1 / 2}} \\
\exp \left(4 H^{\prime}\right) & =\frac{C}{A} \\
\cosh (2 H) & =\frac{2 b c}{\sqrt{A C}}\left(\frac{B^{2}}{4 A C}-\frac{B}{4 b c}-1\right)
\end{aligned}
$$

when the central vertex spins are traced over. One can go in the other direction and decimate the edge Ising spins by replacing the two $R$ interactions and edge field $2 H^{\prime}$ with a single interaction $\beta$ and fields at the end of each edge (i.e. on the vertices). This gives one a standard honeycomb lattice Ising model in field. The relation between the vertex model weights and the Ising parameters $\beta, h$ determined in this manner is identical to that equ.(12).

This decoration-iteration approach strongly supports the observation here that the equivalence between the symmetric 8-vertex model on the honeycomb lattice and the Ising model in field continues to hold for the 4-vertex model on $\Phi^{3}$ random graphs. The decoration iteration transformation is a local transformation which relies only on the valency of each vertex and in both cases all vertices have valency three. It also supports the result that the relation between the Ising and vertex parameters is identical on $\Phi^{3}$ random graphs and the honeycomb lattice, since the decoration-iteration transformation doesn't "see" the randomness, so as far as it is concerned the random and honeycomb lattices are identical f .

The critical behaviour of symmetric vertex models on higher valency regular lattices may be deduced by using the appropriate generalized weak graph transformations to map the models onto Ising models in field in all cases. The generalised weak graph transformation written down by $\mathrm{Wu}$ for general valency $q$

$$
W_{i j}=\frac{1}{\left(1+y^{2}\right)^{q / 2}} \sum_{k=0}^{j}\left(\begin{array}{c}
i \\
k
\end{array}\right)\left(\begin{array}{c}
q-i \\
j-k
\end{array}\right)(-1)^{k} y^{i+j-2 k}
$$

where the vertices are labelled in some suitable order as in the $\Phi^{3}, \Phi^{4}$ cases, is precisely that obtained by picking out the appropriate powers in an expansion of terms such as $(\cos (\theta) X+\sin (\theta) Y)^{q-k}(-\sin (\theta) X+$ $\cos (\theta) Y)^{k}$.

\section{Speculations on Connections with Lorentzian Gravity}

$\Phi^{3}$ graphs are duals to triangulations which are the natural discretisation of Euclidean signature $(++)$ 2 D spacetimes. An analytical continuation to physical Lorentzian $(-+)$ signatures is by no means on such firm ground as the analogous procedure in standard quantum field theory on flat backgrounds. It is therefore of some interest to attempt to formulate discretised theories which might serve as toy models for Lorentzian gravity.

An essential extra ingredient compared with the Euclidean case for any Lorentzian theory is a causal structure. One then faces the option of whether causal structures should be summed over or imposed. A relatively rigid choice was explored in [15] where triangulations with spacelike slices joined by fluctuating

\footnotetext{
${ }^{2}$ One possible fly in the ointment is that loops of length one and two, i.e. double edges ("bubbles") and self-loops ("tadpoles"), are in principle present in the random graphs. The star-triangle relation would degenerate at vertices adjacent to such edges. The simplest solution, which we have taken, is to restrict the ensemble of random graphs to exclude such configurations, which does not affect the critical behaviour of the Ising model though non-universal quantities such as the critical temperature may be changed.
} 
timelike edges were used to triangulate Lorentzian space. It was found that only when branchings were allowed was the critical behaviour of Euclidean gravity recovered upon analytical continuation back.

Another possibility is to postulate that global, or at least long-range, causal structure might emerge dynamically in much the same way that the fractal structure of the 2D manifolds does in the Euclidean case. Inspired by [16, 17] we introduce local causal structure by using spacelike edges to triangulate our Lorentzian spacetime. The normals to all the triangles are then time-like, which can be either past or future directed. It is then natural to return to the dual spin network picture, which gives an embedded directed $\Phi^{3}$ graph if we denote the causal relations between points in the interior of adjacent triangles by arrows pointing from the past to the future of each vertex. This should be contrasted with Euclidean 2D triangulations where the dual picture is of undirected $\Phi^{3}$ graphs.

It is thus natural to use arrow vertex models in this context, rather than the bond vertex models considered so far. However the translation between arrows and bonds turns out to be trivial, as we see below. The various sorts of 3 -valent $\left(\Phi^{3}\right)$ arrow vertices are most conveniently labelled by their (indegree, outdegree). The different possibilities are $(3,0),(0,3),(2,1)$ and $(1,2)$ as shown in Fig.4. A natural choice is to take conjugate weights for the $(3,0)$ and $(0,3)$ vertices and similarly for the $(2,1)$ and $(1,2)$ vertices as this preserves the symmetry under arrow reversal. The partition function for this 4 -arrow-vertex model is thus of the form

$$
Z=\sum_{\Phi_{N}} \sum_{G} a^{N_{1}} \bar{a}^{N_{2}} b^{N_{3}} \bar{b}^{N_{4}}
$$

where the first sum is again over different planar $\Phi^{3}$ graphs, the second over arrow assignments and we have $N_{1}(3,0)$ vertices, $N_{2}(0,3)$ vertices etc. Note that only the $(2,1)$ and $(1,2)$ vertices can be considered as regular spacetime points since the $(3,0)$ and $(0,3)$ vertices act as microscopic black and white holes respectively.

We can obtain this partition function from the $N$ vertex term in the expansion of the free energy of the complex matrix model with the action

$$
S=\frac{1}{2} \operatorname{Tr}\left\{\Phi^{\dagger} \Phi-\frac{\alpha}{3}\left[\left(\Phi^{\dagger}\right)^{3}+\Phi^{3}\right]-i \frac{\beta}{3}\left[\left(\Phi^{3}-\left(\Phi^{\dagger}\right)^{3}\right]-\gamma\left[\left(\Phi^{\dagger}\right)^{2} \Phi+\Phi^{2}\left(\Phi^{\dagger}\right)\right]-i \delta\left[\left(\Phi^{\dagger}\right)^{2} \Phi-\Phi^{2}\left(\Phi^{\dagger}\right)\right]\right\}\right.
$$

where $\Phi$ is now an $M \times M$ complex matrix and $a=\alpha+i \beta, b=\gamma+i \delta$. We can transform simply to a bond vertex formulation of the model by splitting $\Phi$ into Hermitian components $X+i Y$. This allows us to interpret $Y$ edges as those containing bonds and $X$ edges as empty (or vice-versa). The resulting action in terms of $X, Y$

$$
S=\operatorname{Tr}\left\{\frac{1}{2}\left(X^{2}+Y^{2}\right)-\frac{(\alpha+3 \gamma)}{3} X^{3}-\frac{(\beta+3 \delta)}{3} Y^{3}-(\gamma-\alpha) X Y^{2}-(\delta-\beta) X^{2} Y\right\} .
$$

is now clearly that of our 4-bond-vertex model on $\Phi^{3}$ random graphs. This displays Ising criticality, as we have seen, so a suitable tuning of the vertex couplings in equ.(28) (and hence in the original arrow vertex model of equ.(27) ) can reach this point 3 . Since this is a continuous transition one might hope that the diverging correlations of the Ising model at the critical point might be translated back to the appearance of long range casual structure ex nihilo in the arrow vertex model formulation. However, one can see heuristically that closed loops of arrows are present at all scales at the transition point. Cluster algorithms exist for vertex models [18] which act by identifying such closed loops and flipping them. These are effective at combatting critical slowing down so they are identifying and flipping loops at all scales near a continuous transition point. The loops are innocuous from the statistical mechanical point of view, but they represent closed timelike loops when the vertex arrows are interpreted as giving the causal structure.

A profusion of closed timelike loops is obviously an undesirable property and we thus conclude that the particular vertex model discussed here is probably not a suitable candidate for modeling discretised Lorentzian gravity as it stands. It would be interesting to determine what modifications might make it so.

\footnotetext{
${ }^{3}$ Interestingly, the presence of the black hole and white hole vertices appears to be necessary to do this, there is insufficient freedom with only the regular vertices.
} 


\section{Discussion}

We have solved a bond 4-vertex model on an ensemble of planar $\Phi^{3}$ graphs by taking our lead from the honeycomb lattice solution of the symmetric 8-vertex model and exploiting its equivalence to an Ising model in field. An important ingredient of the solution was an orthogonal rotation in the matrices use to define the random graph model in order bring a generic weight configuration on to the Ising locus. This turned out to be functionally identical to the generalised weak graph transformation used in the original honeycomb lattice solution [10]. The method of solution and the equivalence of the matrix rotation to the generalised weak graph transformation on an equivalent regular lattice were also shown to work for higher valency symmetric vertex models on random graphs.

We have seen that the relations between the vertex model parameters and the Ising parameters were identical for the honeycomb and random graph models. This is not so surprising at it might first seem when viewed in the light of the decoration-iteration solution on the honeycomb lattice, since this depends only on the valency of the vertices, which is identical in the random case (modulo caveats about tadpoles and bubbles discussed previously). This result could be couched as a non-renormalization theorem for the appropriate ratios of vertex weights, since putting the models on ensembles of random graphs is equivalent to coupling them to $2 \mathrm{D}$ quantum gravity.

The methods discussed in this paper are restricted to symmetric vertex models since the orthogonal rotation of equ.(5) necessarily gives only symmetric weights when applied to Ising actions such as those in equs.(2, 15,19). From the matrix model point of view the vertex model solution shows that an apparently hopeless potential containing $X^{3}, Y^{3}, X^{2} Y$ and $X Y^{2}$ terms can still give rise to a soluble model, once the matrices are appropriately transformed. Finally, we have speculated on the relation between vertex models and Lorentzian signature gravity, pointing out a potential problem with closed timelike loops when employing the class of models considered here.

\section{Acknowledgements}

This work was partially supported by a Leverhulme Trust Research Fellowship and a Royal Society of Edinburgh/SOEID Support Research Fellowship.

\section{References}

[1] V. Kazakov, Phys. Lett. A119 (1986) 140;

D. Boulatov and V. Kazakov, Phys. Lett. B186 (1987) 139.

[2] Z. Burda and J. Jurkiewicz, Acta Physica Polonica B20 (1989) 94.

[3] V. Kazakov, Mod.Phys.Lett. A4 (1989) 1691; in Seillac Symposium (1987) 93.

[4] B. Eynard and C. Kristjansen, Nucl. Phys. B516 (1998) 529; ibid B455 (1995) 577;

B. Eynard and J. Zinn-Justin, Nucl. Phys. B386 (1992) 558;

B. Duplantier and I. Kostov, Phys. Rev. Lett. 61 (1988) 1433;

I. Kostov, Mod. Phys. Lett. A4 (1989) 217;

M. Gaudin and I. Kostov, Phys. lett. B220 (1989) 200.

[5] V. Knizhnik, A. Polyakov and A. Zamolodchikov, Mod. Phys. Lett. A3 (1988) 819;

F. David, Mod. Phys. Lett. A3 (1988) 1651;

J. Distler and H. Kawai, Nucl. Phys. B321 (1989) 509.

[6] E. Brezin, C. Itzykson, G. Parisi and J. Zuber, Commun. Math. Phys. 59 (1978) 35

M. Mehta, Commun. Math. Phys. 79 (1981) 327. 
[7] P. Ginsparg, "Matrix Models and 2D Gravity" hep-th/9112013, also in Phys. Rep. 254 (1995).

[8] D. Johnston and P. Plechac, Phys. Lett. A248 (1998) 37.

[9] "Two-Matrix model with ABAB interaction", V. Kazakov and P. Zinn-Justin, [ hep-th/9808043].

[10] F. Wu, J. Math. Phys. 15 (1974) 687;

F. Wu, X. Wu and H. Blöte, Phys. Rev. Lett. 62 (1989) 2773;

X. Wu and F. Wu, J. Phys. A22 (1989) L55.

[11] X. Wu and F. Wu, J. Stat. Phys. 50 (1989) 41.

[12] F. Wu, J. Phys. A23 (1990) 375.

[13] F. Wegner, Physica 68 (1973) 570;

J. Nagle, J. Math. Phys. 8 (1968) 1007.

[14] S. Carroll, M. Ortiz and W. Taylor, Nucl.Phys. B468 (1996) 383; Nucl.Phys. B468 (1996) 420; Phys.Rev.Lett. 77 (1996) 3947;

D. Johnston, Phys. Lett. B314 (1993) 6.

[15] J. Ambjørn, and R. Loll, Nucl.Phys. B536 (1998) 407;

"A new perspective on matter coupling in 2d quantum gravity", J. Ambjørn, K. Anagnostopoulos and R. Loll, hep-th/9904012;

J. Ambjørn, J. Nielsen and R. Loll, Chaos Solitons Fractals 10 (1999) 177.

[16] F. Markopoulou, L. Smolin, Phys.Rev. D58, (1998) 084032.

[17] "Dual formulation of spin network evolution", F. Markopoulou, gr-qc/9704013

[18] H. Evertz, G. Lana and M. Marcu, Phys.Rev.Lett. 70 (1993) 875;

H. Evertz and M. Marcu, Nucl. Phys. Proc. Suppl. 30 (1993) 277;

H. Evertz, in "Numerical Methods for Lattice Quantum Many-Body Problems", ed. D.J. Scalapino, Addison Wesley Longman, Frontiers in Physics;

G. Barkema, M. Newman , Phys. Rev. E57, (1998) 1155. 
(a)
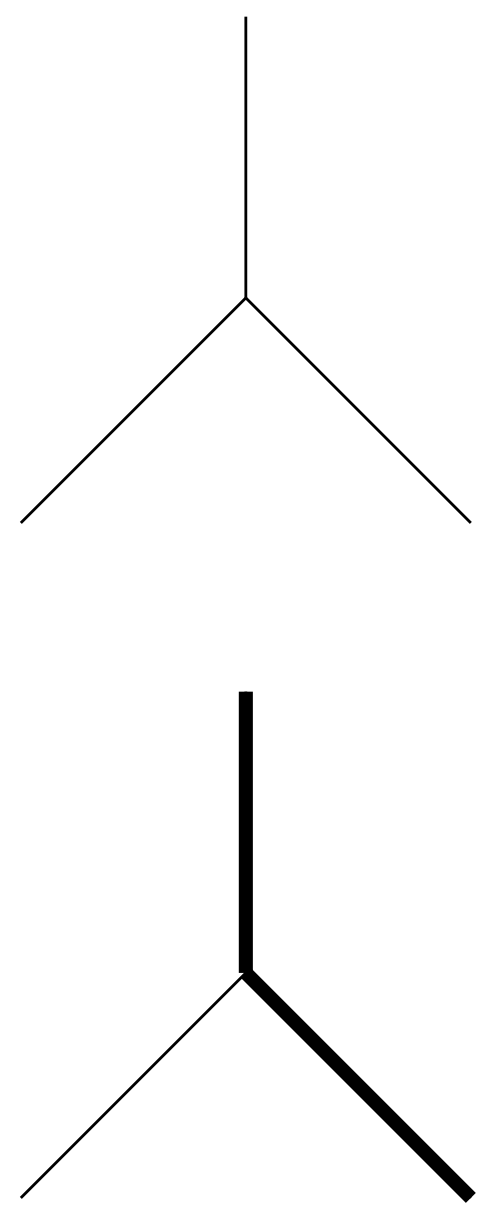

(c) (b)
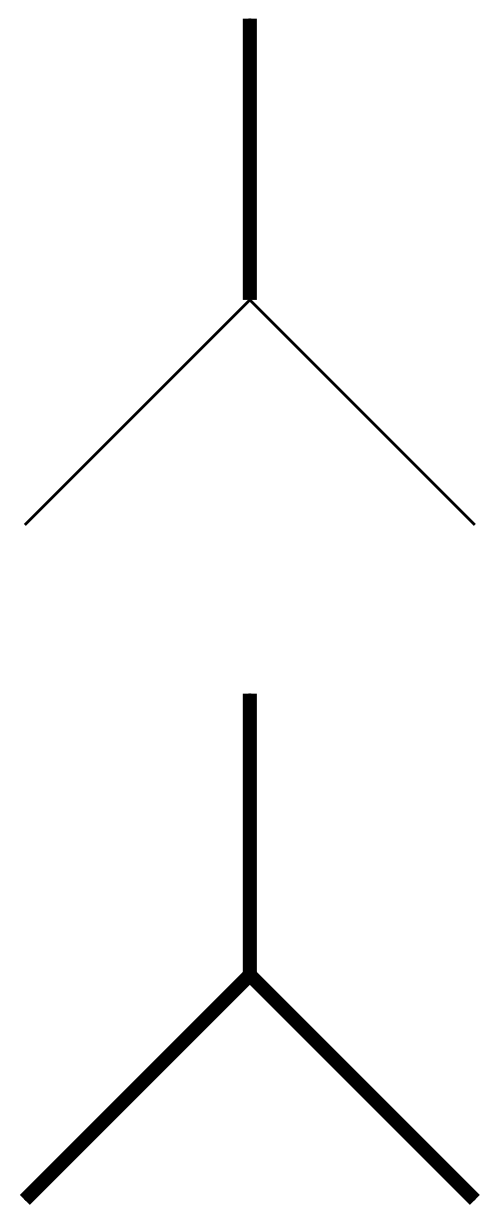

(d)

Figure 1: The possible bond vertices which appear in the model, using the same notation as in [10]. The Ising weights on the random lattice, which can be read off from equ.(可), are: $\tilde{a}=\cosh (h), \tilde{b}=$ $\sinh (h)\left(g^{*}\right)^{1 / 2}, \tilde{c}=\cosh (h) g^{*}$ and $\tilde{d}=\sinh (h)\left(g^{*}\right)^{3 / 2}$. 

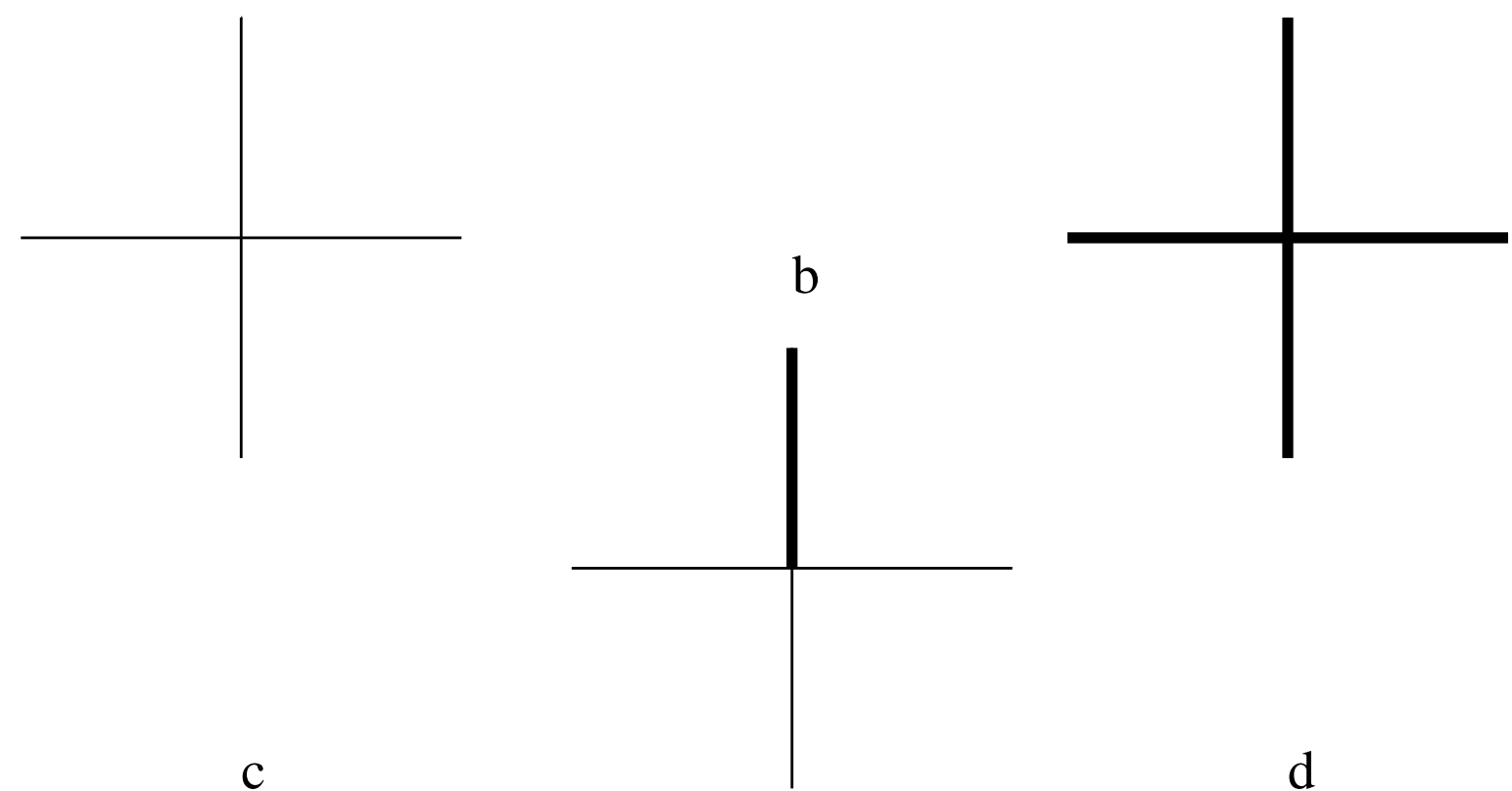

$\mathrm{C}$

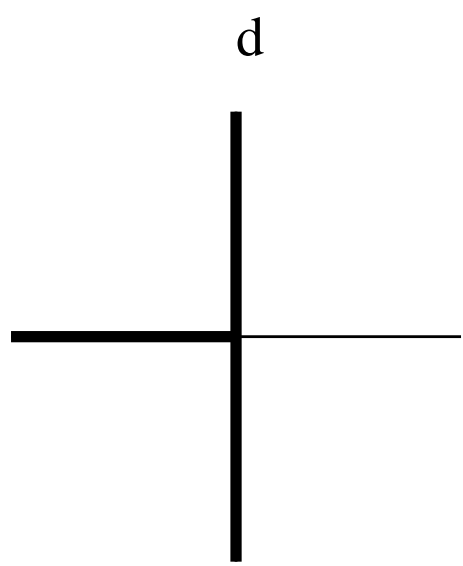

Figure 2: The 5 different vertex weights in the symmetric 16 vertex model on the square lattice. The "straight-through" two bond configuration which is not shown also receives weight $c$. 


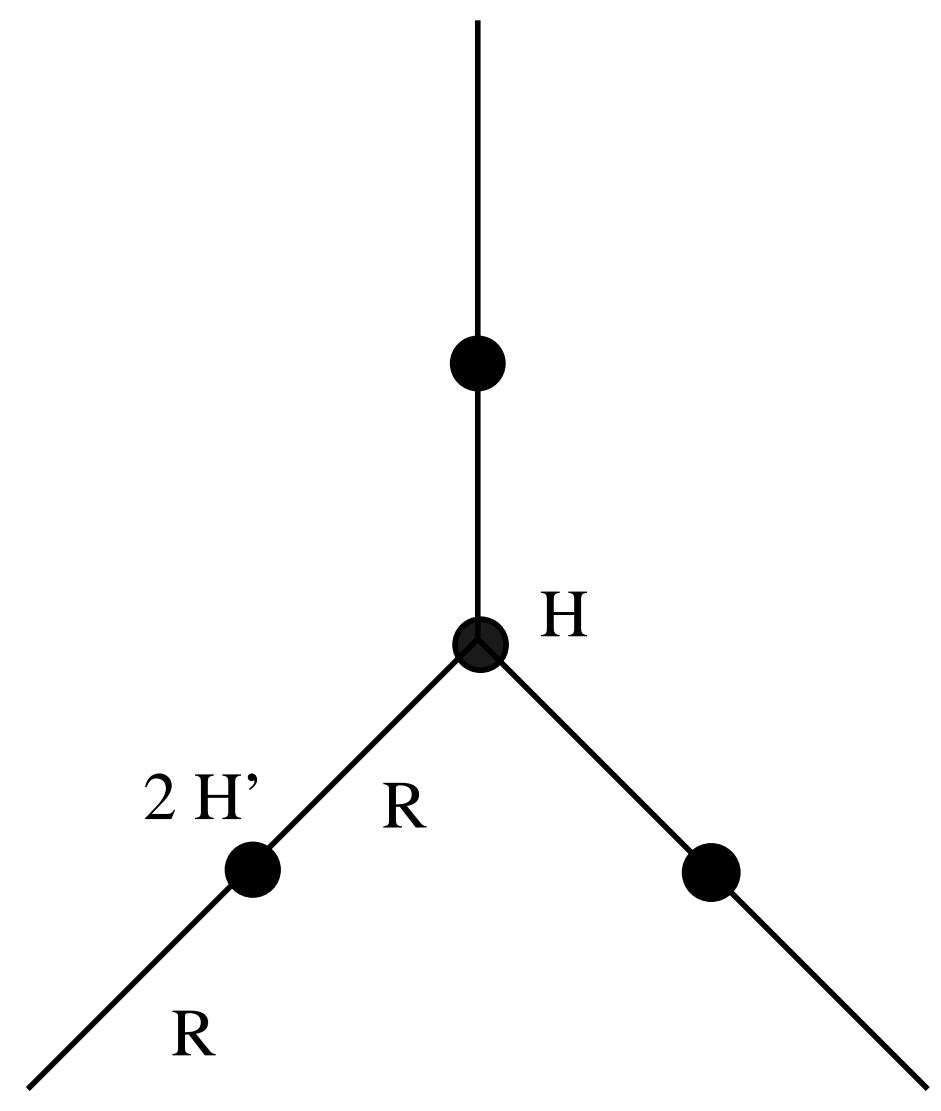

Figure 3: The decorating spins on the edges are subject to an external field $2 H^{\prime}$, the central spin to a field $H$. The edge-vertex spin interactions have weight $R$. 
(a)
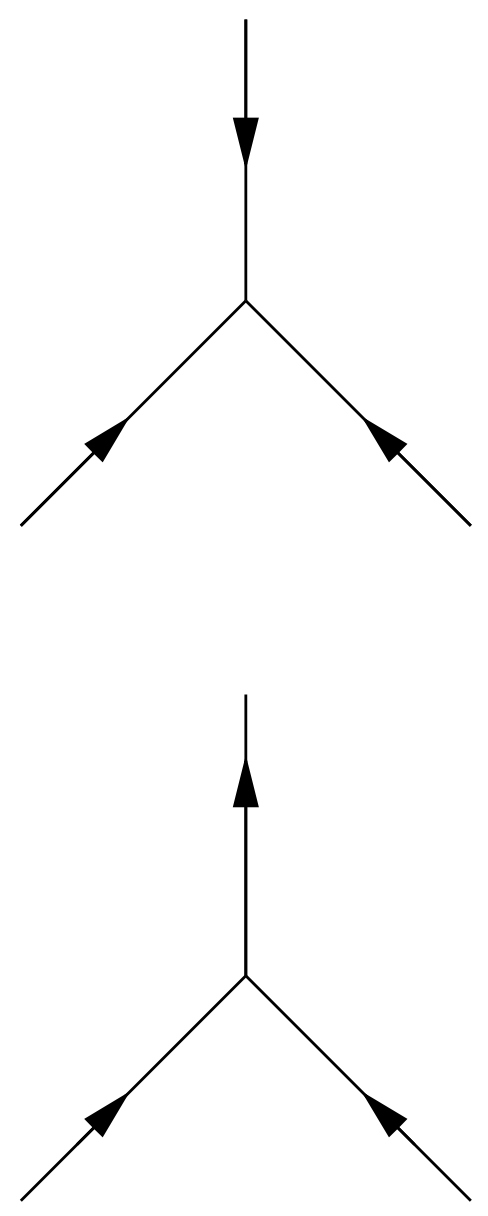

(c) (b)
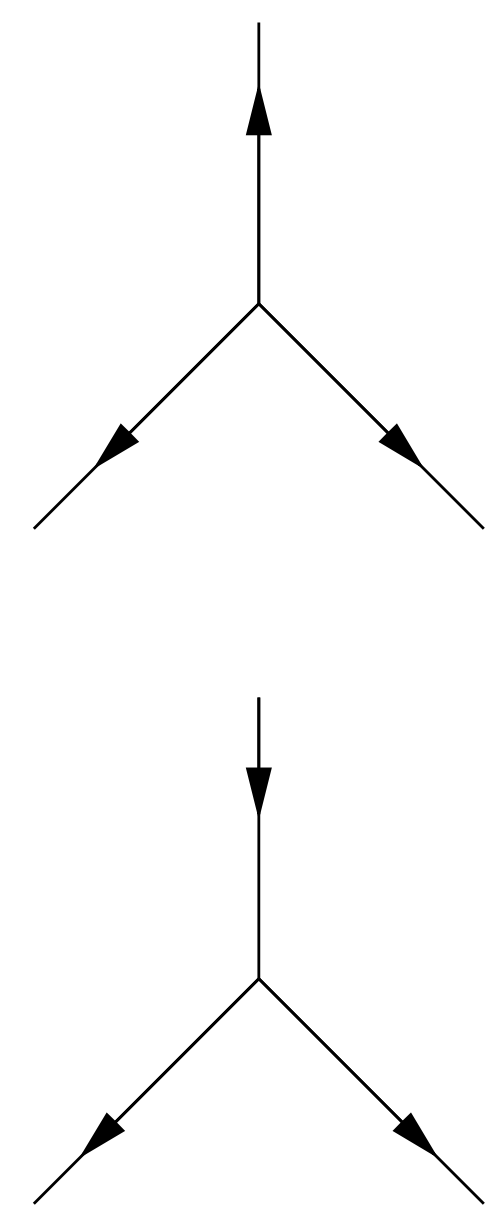

(d)

Figure 4: The possible arrow vertices which appear in the model. Their weights are equivalent to linear combinations of the bond vertex weights in Fig.1. On the random lattices the corresponding terms in the action are :(a) $\left(\Phi^{\dagger}\right)^{3}$, (b) $\Phi^{3}$, (c) $\left(\Phi^{\dagger}\right)^{2} \Phi$, (d) $\Phi^{2} \Phi^{\dagger}$ 